\title{
Enhancing the reach of INO-ICAL using correlated muon and hadron information
}

\author{
Moon Moon Devi ${ }^{* a}$, Tarak Thakore ${ }^{b}$, Sanjib Kumar Agarwalla ${ }^{c}$ and Amol Dighe ${ }^{d}$ \\ ${ }^{a}$ Weizmann Institute of Science, Rehovot 7610001, Israel \\ ${ }^{b}$ Louisiana State University, Baton Rouge, Louisiana 70803, USA \\ ${ }^{c}$ Institute of Physics, Sachivalaya Marg, Sainik School Post, Bhubaneswar 751005, India \\ ${ }^{d}$ Tata Institute of Fundamental Research, Mumbai 400005, India \\ E-mail: mmdevi@weizmann.ac.il, thakore@phys.lsu.edu, \\ sanjibeiopb.res.in, amoletifr.res.in
}

\begin{abstract}
The magnetized iron calorimeter (ICAL) at the India-based neutrino observatory (INO) aims at distinguishing the neutrino mass hierarchy as well as determining the atmospheric neutrino parameters with a fine precision. The ICAL can detect muons with good reconstruction efficiency and momentum $\left(E_{\mu}, \cos \theta_{\mu}\right)$ resolution. It is also capable of measuring the hadron energy $E_{\text {had }}^{\prime} \equiv$ $E_{v}-E_{\mu}$ by calibrating the hadron shower hits. For a given neutrino event, the correlation between $E_{\text {had }}^{\prime}$ and $E_{\mu}$ is an important property, which may be used for improving the oscillation parameter estimation. We take care of this correlation by binning the events in $\left(E_{\mu}, \cos \theta_{\mu}, E_{h a d}^{\prime}\right)$. A $\chi^{2}$ analysis is performed after incorporating the ICAL muon and hadron response, obtained from GEANT4 simulation. We show that, with an exposure of $500 \mathrm{kt}$-year, the ICAL can rule out the wrong hierarchy with a $\Delta \chi^{2} \approx 9$, which marks an enhancement of about $40 \%$ compared with the muon-only analysis. The inclusion of hadron information also improves the precision bounds on $\left|\Delta m_{32}^{2}\right|, \theta_{23}$ and its octant. We show that, 10 years of ICAL exposure would be able to measure $\sin ^{2} \theta_{23}$ and $\left|\Delta m_{32}^{2}\right|$ to a relative $1 \sigma$ precision of $12 \%$ and $2.9 \%$ respectively.
\end{abstract}

16th International Workshop on Neutrino Factories and Future Neutrino Beam Facilities - NUFACT2014, 25 -30 August, 2014

University of Glasgow, United Kingdom

${ }^{*}$ Speaker. 


\section{Introduction}

The Atmospheric neutrinos, which offer a wide range of energy and baseline, can play a crucial role in the study of the neutrino oscillations. The neutrino mass hierarchy $(\mathrm{MH})$ (the normal mass hierarchy (NH): $m_{1}<m_{2}<m_{3}$, the inverted hierarchy (IH): $m_{3}<m_{1}<m_{2}$ ), can possibly be obtained by probing the matter effects on neutrinos as they propagate through the Earth [1]. The matter effects experienced by the neutrinos and the antineutrinos are different, and separate study of these effects is important for the mass hierarchy identification [2]. The atmospheric neutrinos passing through the Earth experience significant matter effects and thus they can be used to discover the true mass ordering. In the $\mathrm{GeV}$ energy range, the atmospheric neutrino flux is small, which necessitates very large detectors.

The magnetized Iron Calorimeter detector (ICAL), with a mass of about $50 \mathrm{kt}$, at the Indiabased Neutrino Observatory (INO) is designed to study neutrino mixing parameters using atmospheric neutrinos [3]. The primary goals of the experiment are the identification of neutrino mass hierarchy and the precision measurements of atmospheric oscillation parameters $\left(\sin ^{2} \theta_{23},\left|\Delta m_{32}^{2}\right|\right)$. The underground lab facility for the experiment is being constructed at Theni in Southern India. The ICAL setup consists of 151 layers of $5.6 \mathrm{~cm}$ thick iron plates interspersed with Resistive Plate Chambers (RPCs) as active detector elements [4].

The detector is primarily optimized to measure the muon momentum with high efficiency in the $\mathrm{GeV}$ range. Further, the ICAL, being a magnetized detector, would also be able to distinguish between the $v_{\mu}$ and $\bar{v}_{\mu}$ events, through the separate identification of the muon and antimuon tracks in the magnetic field. In addition, it is also capable of detecting of the hadrons produced in the $v_{\mu}$ interactions and measuring their energy, though with a coarser resolution. The initial analysis of the physics potential of ICAL was performed using the muon momentum $\left(E_{\mu}, \cos \theta_{\mu}\right)$ only $[5,6]$. Although the hadron energy is measured with relatively poorer resolutions, it provides crucial information on the event, and may be utilized when used along with the muon data. Studies showed that by using inelasticity parameter, i.e., $y \equiv\left(E_{v}-E_{\mu}\right) / E_{v}=E_{\mathrm{had}}^{\prime} / E_{v}$ in each event, the $\mathrm{MH}$ reach can be improved by $20-50 \%$ [7]. The $y$ is approximately the fraction of the neutrino energy carried by hadrons. Note that the correlation between the muon and the hadrons in each event is important for such study. In order to use the information on the hadron energy and their correlation with the muons, we use the muon energy $\left(E_{\mu}\right)$, muon direction $\left(\cos \theta_{\mu}\right)$ and hadron energy $\left(E_{\text {had }}^{\prime}=E_{v}-E_{\mu}\right)$ from each event as the observables .

We present here the analysis and results of the statistical approach to find the ICAL sensitivity with correlated muon and hadron information [8]. The enhancement in the detector's potential as a result of the addition of the hadron information is highlighted. The ICAL response to the muon and hadrons, and the hadronic contribution in the events of interest are discussed in section 2. The analysis procedure is described in section 3. We present the ICAL sensitivity to the MH determination and the precision measurements of $\left|\Delta m_{32}^{2}\right|, \sin ^{2} \theta_{23}$ and its octant in section 4 .

\section{Neutrino interactions and event reconstruction in ICAL}

The atmospheric $v_{\mu}$ and $\bar{v}_{\mu}$ interact with the iron target through quasi-elastic (QE) and resonance scattering (RS) and deep inelastic scattering (DIS) processes as well as a negligible fraction 

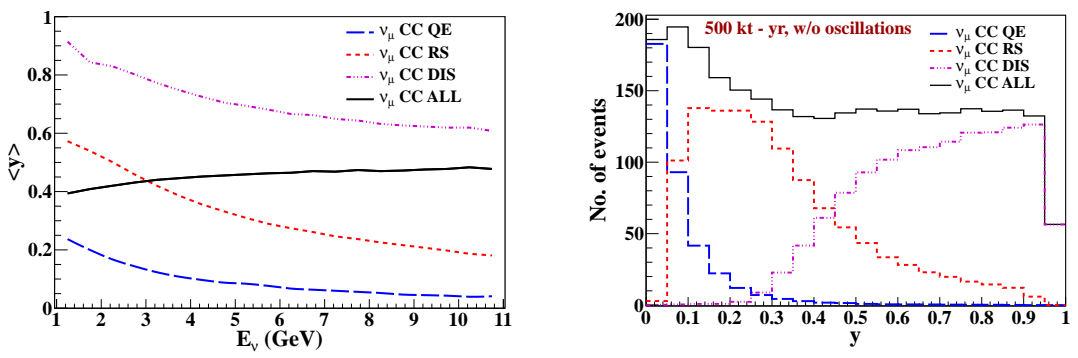

Figure 1: The average inelasticities $\langle y\rangle$ in the three processes, as functions of the neutrino energies (left). The distribution of inelasticity in events with neutrino energies in the range $4-7 \mathrm{GeV}$, with an exposure of $500 \mathrm{kt}-\mathrm{year}$, in the absence of oscillations (right). [8]

of diffractive and coherent processes. In the $\mathrm{CC}$ interactions, a muon is produced in the final state. In the sub- $\mathrm{GeV}$ neutrino energy range, the $\mathrm{QE}$ process dominates, where no hadrons are produced and the final state muon carries most of the available energy As the energy increases RS and DIS processes start dominating and at a few GeVs DIS becomes the most prominent process. The RS events typically contain a single pion in the final state, though in a small fraction of events there are multiple pions. The DIS events produce multiple hadrons.

The ICAL response to muons and hadrons are obtained using a GEANT4-based detector simulation algorithm $[6,9,10]$. We term the passage of a charged particle as detected in the RPCs as hits. The RPCs provide the (X, Y) coordinates of the hits, while the Z-coordinate is calculated from the RPC layer number. The muon hits form track-like features, while hadron hits produce showers. The muon energy and direction are reconstructed using a Kalman Filter-based track reconstruction algorithm. The hadron energy is parametrized in terms of $E_{\mathrm{had}}^{\prime} \equiv E_{v}-E_{\mu}$, and is calibrated against the number of hadron hits in each event.

The importance of the hadron energy in an event can be understood from the inelasticity $y$ in an event. The average inelasticities $\langle y\rangle$ in the three kinds of processes, as functions of neutrino energies, have been shown in the left panel of Fig. 1. Clearly, $\langle y\rangle$ in the DIS events is significant, which implies that a large fraction of the incoming neutrino energy goes into hadrons in the energy range of interest for $\mathrm{MH}$ determination. While $\langle y\rangle$ does not fluctuate much over this energy range, the inelasticities in individual events have a wide distribution (see the right panel of Fig. 1). Hence it is important to consider the $y$ values in individual events. In the analysis procedure, which is discussed in section 3, the energies of hadrons and muons obtained in each event are used as separate variables so that the correlation between them is preserved.

\section{The analysis procedure}

The analysis procedure [8] is discussed below.

1. Event Generation: The generator NUANCEv3.5 [11], with the ICAL specifications as input, is used as the Monte Carlo event generator to produce atmospheric neutrino interactions. The atmospheric neutrino flux provided by Honda et al. at the Super Kamiokande site is used [12]. In order to minimize the statistical fluctuations, $\mathrm{CC} v_{\mu}$ events are produced for a large exposure of $50000 \mathrm{kt}-\mathrm{yr}$ and then scaled down to the required exposures. Producing events 
Table 1: Benchmark oscillation parameters used in this analysis [13].

\begin{tabular}{|c|c|c|}
\hline Parameter & True value & Marginalization range \\
\hline $\sin ^{2} 2 \theta_{13}$ & $0.09,0.1,0.11$ & {$[0.07,0.11]$} \\
\hline $\sin ^{2} \theta_{23}$ & $0.4,0.5,0.6$ & {$[0.36,0.66]$} \\
\hline$\Delta m_{e f f}^{2} / \mathrm{eV}^{2}$ & $\pm 2.4 \times 10^{-3}$ & $\begin{array}{c}{[2.1,2.6] \times 10^{-3}(\mathrm{NH})} \\
-[2.6,2.1] \times 10^{-3}(\mathrm{IH})\end{array}$ \\
\hline $\sin ^{2} 2 \theta_{12}$ & 0.84 & Not marginalized \\
\hline$\Delta m_{21}^{2} / \mathrm{eV}^{2}$ & $7.5 \times 10^{-5}$ & Not marginalized \\
\hline$\Delta_{c p}$ & $0^{\circ}$ & Not marginalized \\
\hline
\end{tabular}

with such a large exposure at all possible sets of the oscillation parameters is practically impossible, so the event generator is run only once for un-oscillated neutrino flux, and later a re-weighting algorithm is used to incorporate the oscillations.

2. Inclusion of the oscillations and detector response: The events are re-weighted using a random selection algorithm. The survival/ oscillation probabilities for the certain channel is calculated using the given set of oscillation parameters. The benchmark values of the oscillations parameters are used as in [13]. Then for each event, a uniform random number $R$ between 0 and 1 is generated and compared to the probability to pick/discard that event. The re-weighted events are then binned in the observables $\left(E_{\mu}, \cos \theta_{\mu}, E_{\text {had }}^{\prime}\right)$ and folded with the ICAL response. The ICAL lookup tables for both muon and hadron responses are used $[6,9,10]$. First, the events in each bin were multiplied by the muon reconstruction efficiency and CID efficiency. Then the integrals of the detector response functions of the observables are evaluated, and using them the measured distribution of the events are obtained.

3. The $\chi^{2}$ analysis: For the $\chi^{2}$ analysis the events are re-distributed in wider and non-uniform bins, the bin widths being comparable to the respective resolutions. A scheme of $20 E_{\mu}$ bins in the range $(1-11) \mathrm{GeV}, 21 \cos \theta_{\mu}$ bins in the range $[-1,+1]$, and $4 E_{\mathrm{had}}^{\prime}$ bins in the range $(0-15) \mathrm{GeV}$ are used for each polarity of muon.

The Poissonian $\chi_{ \pm}^{2}$ for events with a $\mu^{ \pm}$is defined as

$$
\chi_{ \pm}^{2}=\min _{\xi_{l}} \sum_{i=1}^{N_{E_{\text {had }}^{\prime}}} \sum_{j=1}^{N_{E_{\mu}}} \sum_{k=1}^{N_{\cos \theta_{\mu}}}\left[2\left(N_{i j k}^{\text {theory }}-N_{i j k}^{\mathrm{data}}\right)-2 N_{i j k}^{\mathrm{data}} \ln \left(\frac{N_{i j k}^{\text {theory }}}{N_{i j k}^{\mathrm{data}}}\right)\right]+\sum_{l=1}^{5} \xi_{l}^{2} .
$$

In the analysis, the following five systematic errors are included using the method of pulls [14] : (i) Flux normalization error (20\%), (ii) cross-section error (10\%), (iii) tilt error (5\%), (iv) zenith angle error (5\%), and (v) overall systematics (5\%). The total $\chi^{2}$ is obtained by adding the individual contributions from $\mu^{-}$and $\mu^{+}$events and a $8 \%$ prior (at $1 \sigma$ ) on $\sin ^{2} 2 \theta_{13}$ :

$$
\chi_{\text {ICAL }}^{2}=\chi_{-}^{2}+\chi_{+}^{2}+\chi_{\text {prior }}^{2} .
$$

This $\chi_{\text {ICAL }}^{2}$ is marginalized over the pull variables $\xi_{l}$ and over the $3 \sigma$ allowed range of the relevant oscillation parameters. 


\section{Results with the $\left(E_{\mu}, \cos \theta_{\mu}, E_{\mathrm{had}}^{\prime}\right)$ analysis}

The results are discussed here. We begin with the enhancement obtained in the MH sensitivity.

\subsection{Sensitivity to the neutrino mass hierarchy}

The statistical significance of the analysis to discard the wrong hierarchy is quantified by

$$
\Delta \chi_{\mathrm{ICAL}-\mathrm{MH}}^{2}=\chi_{\mathrm{ICAL}}^{2}(\text { false } \mathrm{MH})-\chi_{\mathrm{ICAL}}^{2}(\text { true } \mathrm{MH}),
$$

where $\chi_{\text {ICAL }}^{2}$ (true $\left.\mathrm{MH}\right)$ and $\chi_{\text {ICAL }}^{2}($ false $\mathrm{MH})$ are obtained from a fit to the observed data assuming certain true and false mass hierarchy, respectively. With the statistical fluctuations suppressed, $\chi_{\text {ICAL }}^{2}($ true $\mathrm{MH}) \approx 0$. The statistical significance has also been represented in terms of $n \sigma$, where $n \equiv \sqrt{\Delta \chi_{\text {ICAL-MH }}^{2}}$. This relation provides the median sensitivity in the frequentist approach of hypothesis testing.

In Fig. 2 the distributions of $\Delta \chi_{-}^{2} \equiv \chi_{-}^{2}(\mathrm{IH})-\chi_{-}^{2}(\mathrm{NH})$ in the reconstructed $E_{\mu}-\cos \theta_{\mu}$ plane are shown. The left panel shows the results for the analysis that does not use the hadron energy information, while the right panel shows the analysis where events are further divided into four sub-bins of $E_{\text {had }}^{\prime}$ and for each $E_{\mu}-\cos \theta_{\mu}$ bin, the $\Delta \chi_{-}^{2}$ has been summed over the hadron energy bins. Similar improvement can be observed for the antineutrinos. It can be observed that, with the addition of the hadron energy information, the area in the $E_{\mu}-\cos \theta_{\mu}$ plane that contributes significantly to $\Delta \chi_{ \pm}^{2}$ increases, which in turn improves the net $\Delta \chi_{ \pm}^{2}$. This increase in $\chi_{ \pm}^{2}$ is contributed by not only the information contained in the hadron energy measurement, but also the correlation between the hadron energy and muon momentum. Note that, the constant contribution in $\chi^{2}$ coming from the term involving the five pull parameters $\xi_{l}^{2}$ in Eq. (3.1) has not been considered. Also, the marginalization over the oscillation parameters in the fit has not been performed here. However the MH sensitivity results are obtained with the full pull contributions and marginalizations.
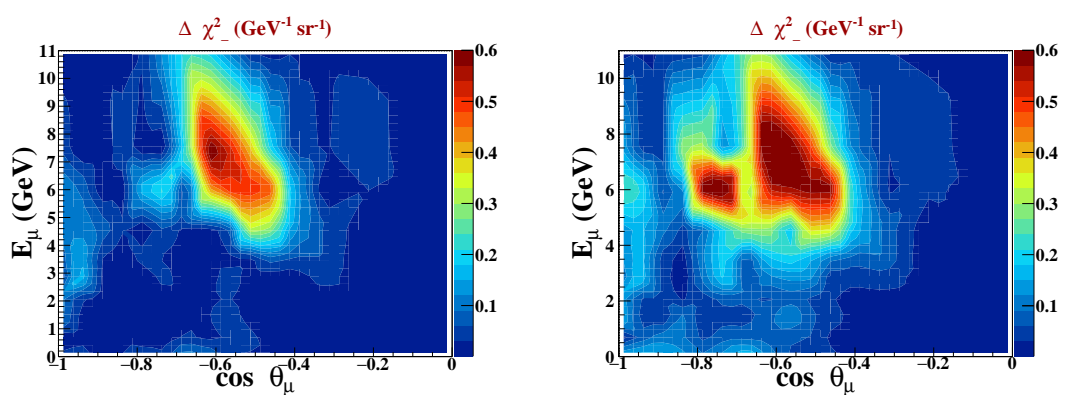

Figure 2: The distribution of $\Delta \chi_{-}^{2}$ per unit area in $\left(E_{\mu}-\cos \theta_{\mu}\right)$ plane, without (left) and with (right) hadron information. The $\mathrm{NH}$ is assumed to be the true hierarchy, and $500 \mathrm{kt}-\mathrm{year}$ of ICAL exposure is used. [8]

The comparison of the $\Delta \chi_{\text {ICAL-MH }}^{2}$ obtained in this analysis with that from the muon-only analysis is shown in Fig. 3, as a function of the ICAL run-time for true NH. After including the $E_{\text {had }}^{\prime}$ information, 10 years of running can rule out the wrong hierarchy with $\Delta \chi_{\text {ICAL-MH }}^{2} \approx 9.5$ (for true $\mathrm{NH}$ ), and $\Delta \chi_{\mathrm{ICAL}-\mathrm{MH}}^{2} \approx 8.7$ (for true $\mathrm{IH}$ ), which mark an improvement of about $40 \%$ over the muon-only analysis. Fig. 4 shows the range of $\Delta \chi_{\text {ICAL-MH }}^{2}$ for different true $\sin ^{2} \theta_{23}$. 

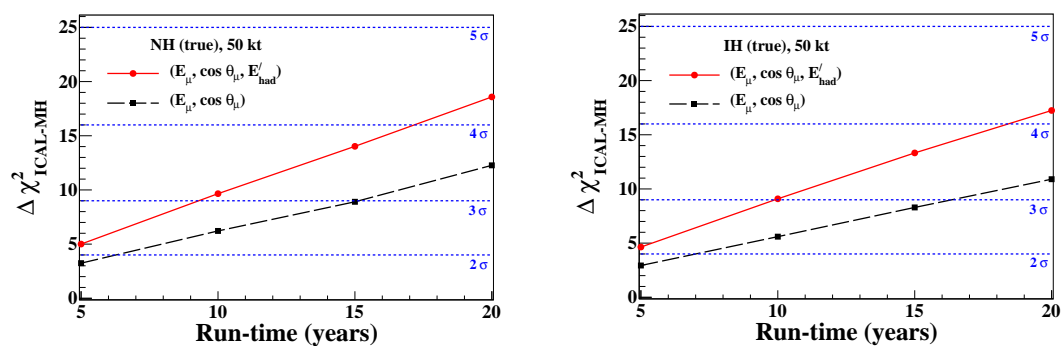

Figure 3: The $\Delta \chi_{\text {ICAL-MH }}^{2}$ as a function of the run-time assuming NH (left) and IH (right) as true hierarchy. The red line shows the results with hadron information, while the black dashed line shows the same without including hadron information. [8]
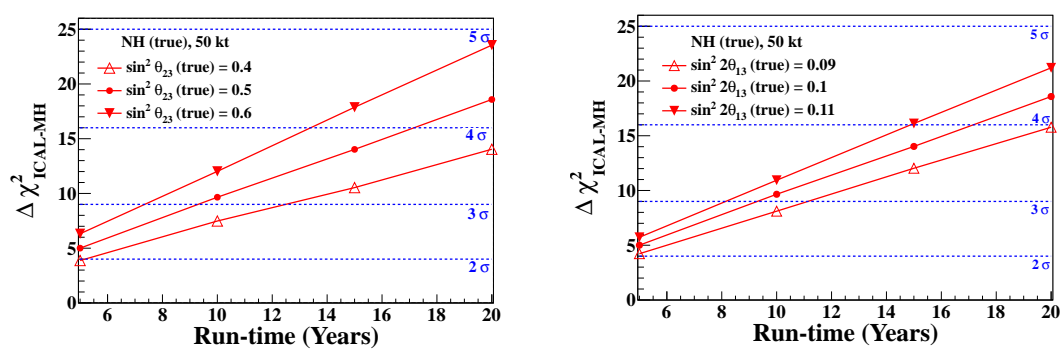

Figure 4: The variation of mass hierarchy sensitivity to true values of $\theta_{23}$ (left) and $\theta_{13}$ (right), for true $\mathrm{NH}$, as a function of the run-time of $50 \mathrm{kt} \mathrm{ICAL.} \mathrm{[8]}$

\subsection{Precision measurements of $\left|\Delta m_{32}^{2}\right|$ and $\sin ^{2} \theta_{23}$}

The precision in the measurements of the parameter $\lambda\left(\lambda=\sin ^{2} \theta_{23}\right.$ or $\left.\left|\Delta m_{32}^{2}\right|\right)$, is quantified as

$$
\Delta \chi_{\mathrm{ICAL}-\mathrm{PM}}^{2}(\lambda)=\chi_{\mathrm{ICAL}-\mathrm{PM}}^{2}(\lambda)-\chi_{0}^{2},
$$

where $\chi_{0}^{2}$ is the minimum value of $\chi_{\text {ICAL-PM }}^{2}$ in the allowed parameter range. It is observed that with the inclusion of the information on $E_{\text {had }}^{\prime}, 500 \mathrm{kt}$-year of ICAL exposure would be able to measure $\sin ^{2} \theta_{23}$ to a relative $1 \sigma$ precision of $12 \%$ and $\left|\Delta m_{32}^{2}\right|$ to $2.9 \%$. With the muon-only approach, the same relative precisions were $13.7 \%$ and $5.4 \%$, respectively. The $\sin ^{2} \theta_{23}$ precision depends mainly on the event statistics, which is not changed by the addition of the $E_{\text {had }}^{\prime}$ information, thus only a small difference is observed in the two analyses. The independent measurements of $E_{\mu}$ and $E_{\text {had }}^{\prime}$ lead to a better estimation of $E_{v}$, which appears in the oscillation expression as $\sin ^{2}\left(\Delta m^{2} L / E_{v}\right)$, thus resulting in a significant improvement of the measurement of $\left|\Delta m_{32}^{2}\right|$. The left panel of Fig. 5 shows the comparison of $\Delta \chi_{\text {ICAL-PM }}^{2}\left(\left|\Delta m_{32}^{2}\right|\right)$, with and without hadron energy information. The ICAL $500 \mathrm{kt}$ - year projected reach has been compared to the current results from other experiments in the right panel of Fig. 5.

\subsection{Sensitivity to the octant of $\theta_{23}$}

In analogy with the MH discovery potential, the statistical significance of the analysis to rule out the wrong octant of $\theta_{23}$ is defined as

$$
\Delta \chi_{\mathrm{ICAL}-\mathrm{OS}}^{2}=\chi_{\mathrm{ICAL}}^{2}(\text { false octant })-\chi_{\mathrm{ICAL}}^{2}(\text { true octant }) .
$$





Figure 5: (Left) The $\Delta \chi_{\text {ICAL-PM }}^{2}\left(\left|\Delta m_{32}^{2}\right|\right)$, with and without hadron energy information (left), The true hierarchy is assumed to be NH. (Right) The ICAL 90\% C.L. ( 2 dof) contour in the $\sin ^{2} \theta_{23}-\left|\Delta m_{32}^{2}\right|$ plane. The current limits from Super- Kamiokande [15], MINOS [16], and T2K [17] have also been shown. [8]

It is observed that the potential of distinguishing the $\theta_{23}$ octant with the ICAL data alone is rather weak. A $2 \sigma$ identification of the octant would be possible with the $500 \mathrm{kt}$-year ICAL data alone only when the true hierarchy is $\mathrm{NH}$ and the true octant is $\mathrm{LO}\left(\sin ^{2} \theta_{23}(\right.$ true $\left.)<0.395\right)$.

\section{Summary and concluding remarks}

In this paper we have discussed the motivation, methodology and results of the statistical analysis which is used to assess the ICAL physics potentials with the inclusion of the hadron energy. Though ICAL is primarily optimized for muon detection, its capability of detecting hadrons and estimations their energy has been an additional advantage. The enhancement is not only due to the hadron energy, but also due to the correlation between the hadron energy and the muon momentum in an event. The analysis presented in this chapter uses hadron energy, muon energy and muon direction as separate observables in each event.

Significant improvements have been observed in the ICAL sensitivities, using this analysis. After including the $E_{\text {had }}^{\prime}$ information, 10 years of running can rule out the wrong hierarchy with $\Delta \chi_{\text {ICAL-MH }}^{2} \approx 9.5$ (for true $\mathrm{NH}$ ), and $\Delta \chi_{\text {ICAL-MH }}^{2} \approx 8.7$ (for true $\mathrm{IH}$ ), which mark an improvement of about $40 \%$ over the muon-only analysis. It is observed that with the inclusion of $E_{\text {had }}^{\prime}$ information, $500 \mathrm{kt}$-year of ICAL exposure would be able to measure $\sin ^{2} \theta_{23}$ to a relative $1 \sigma$ precision of $12 \%$ and $\left|\Delta m_{32}^{2}\right|$ to $2.9 \%$. However, that the potential of distinguishing the $\theta_{23}$ octant with the ICAL data alone is rather weak. A $2 \sigma$ identification of the octant is possible with the $500 \mathrm{kt}$-year ICAL data alone only when, the true hierarchy is $\mathrm{NH}$ and the true octant is $\mathrm{LO}\left(\sin ^{2} \theta_{23}\right.$ (true $\left.)<0.395\right)$.

It is clear from the above results that the inclusion of correlated hadron energy information improves oscillation physics sensitivities in almost all areas. Depending on the present status of the ICAL simulations certain assumptions had to be made. As the understanding of the detector improves, those issues can be taken care of, which would also affect the physics reach. However, this paper demonstrates quantitatively that, with the same conditions and assumptions, the inclusion of event-by-event hadron energy information in the analysis increases the reach for mass hierarchy identification and $\left|\Delta m_{32}^{2}\right|$ precision by a significant amount.

This analysis procedure is expected to become the preferred one for future analyses of ICAL physics reach. Thus a better understanding of the hadron response of the detector, and development of efficient algorithms to use the hadron data would be important. 


\section{Acknowledgments}

We would like to acknowledge the INO collaboration for the support during the course of this work. We are also thankful to the NUFACT2014 organizers. MMD acknowledges the Homi Bhabha National Institute for the international travel grant.

\section{References}

[1] S. P. Mikheev et al., Resonance enhancement of oscillations in matter and solar neutrino spectroscopy, Sov. J. Nucl. Phys. 42 (1985) 913-917

[2] A. Y. Smirnov, Neutrino mass hierarchy and matter effects, PoS Neutel 2013 (2014) 027 [arXiv:1312.7309 [hep-ph]].

[3] M. S. Athar et al., [INO Collaboration], INO: Project Report, Volume I. INO-2006-01.

[4] V. M. Datar et al., Development of glass resistive plate chambers for INO experiment, Nucl. Instrum. Meth. A 602 (2009) 744.

[5] A. Ghosh et al., 'Determining the Neutrino Mass Hierarchy with INO, T2K, NOvA and Reactor Experiments,” JHEP 1304, 009 (2013) [arXiv:1212.1305 [hep-ph]].

[6] T. Thakore et al.,The Reach of INO for Atmospheric Neutrino Oscillation Parameters, JHEP 1305 (2013) 058 [arXiv:1303.2534 [hep-ph]].

[7] M. Ribordy and A. Y. Smirnov, Improving the neutrino mass hierarchy identification with inelasticity measurement in PINGU and ORCA, Phys. Rev. D 87 (2013) 113007 [arXiv:1303.0758 [hep-ph]].

[8] M. M. Devi et al., Enhancing sensitivity to neutrino parameters at INO combining muon and hadron information, JHEP 1410 (2014) 189 [arXiv:1406.3689 [hep-ph]].

[9] A. Chatterjee et al., A Simulations Study of the Muon Response of the Iron Calorimeter Detector at the India-based Neutrino Observatory, JINST 9 (2014) P07001 [arXiv:1405.7243 [physics.ins-det]].

[10] M. M. Devi et al., Hadron energy response of the Iron Calorimeter detector at the India-based Neutrino Observatory, JINST 8 (2013) P11003 [arXiv:1304.5115 [physics.ins-det]].

[11] D. Casper, The Nuance neutrino physics simulation, and the future, Nucl. Phys. Proc. Suppl. 112 (2002) 161 [hep-ph/0208030]; see also, http://nuint.ps.uci.edu/nuance/.

[12] M. Honda et al., A New calculation of the atmospheric neutrino flux in a 3-dimensional scheme, Phys. Rev. D 70 (2004) 043008 [astro-ph/0404457].

[13] D. V. Forero et al.,Neutrino oscillations refitted, [arXiv:1405.7540 [hep-ph]]; http://www.nu-fit.org/.

[14] M. C. Gonzalez-Garcia et al., Atmospheric Neutrino Oscillations and New Physics, Phys. Rev. D 70 (2004) 033010 [hep-ph/0404085].

[15] A. Himmel [SK Collaboration], Recent results from Super-Kamiokande, AIP Conf. Proc. 1604 (2014) 345 [arXiv:1310.6677 [hep-ex]].

[16] P. Adamson et al. [MINOS Collaboration], Combined analysis of $v_{\mu}$ disappearance and $v_{\mu} \rightarrow v_{e}$ appearance in MINOS using accelerator and atmospheric neutrinos, Phys. Rev. Lett. 112 (2014) 191801 [arXiv:1403.0867 [hep-ex]].

[17] K. Abe et al. [T2K Collaboration], Precise Measurement of the Neutrino Mixing Parameter $\theta_{23}$ from Muon Neutrino Disappearance in an Off-Axis Beam, Phys. Rev. Lett. 112 (2014) 181801 [arXiv:1403.1532 [hep-ex]]. 